\title{
Difference of Serum Cytokine Profile in Allergic Asthma Patients According to Disease Severity
}

\author{
Lin Sun $^{1-3, *}$, Bo Peng ${ }^{1-3, *}$, Jun Zhou ${ }^{1-3}$, Ping Wang ', Yuqing Mo ${ }^{4}$, Guofang $X u^{\prime}$, Yi Tao', Hejie Song ${ }^{1-3}$, \\ Wei Tang ${ }^{I-3}$, Meiling Jin ${ }^{4}$
}

'Department of Respiratory and Critical Care Medicine, Ruijin Hospital, Shanghai Jiao Tong University School of Medicine, Shanghai, People's Republic of China; ${ }^{2}$ Institute of Respiratory Diseases, Shanghai Jiao Tong University School of Medicine, Shanghai, People's Republic of China; ${ }^{3}$ Shanghai Key Laboratory of Emergency Prevention, Diagnosis and Treatment of Respiratory Infectious Diseases, Shanghai, People's Republic of China; ${ }^{4}$ Department of Pulmonary and Critical Care Medicine, Zhongshan Hospital, Fudan University, Shanghai, People's Republic of China

*These authors contributed equally to this work

Correspondence: Wei Tang, Department of Respiratory and Critical Care Medicine, Ruijin Hospital, Shanghai Jiao Tong University School of Medicine, Shanghai, People's Republic of China, Email tina_tangwei@I63.com; Meiling Jin, Department of Pulmonary and Critical Care Medicine, Zhongshan Hospital, Fudan University, Shanghai, People's Republic of China, Email mljinII8@I63.com

Purpose: Allergic asthma is a heterogeneous disease with complex underlying mechanisms. Cytokines are key mediators in immune system and potential indicators of disease status. The aim of this study is to compare the difference of serum cytokine profile in allergic asthma patients with different disease severity and explore candidate biomarkers for disease monitoring and targeting therapeutic agents.

Patients and Methods: A total of 40 allergic asthmatics (mild, $n=22$; moderate-to-severe, $n=18$ ) were included in this study. Serum samples, lung function and exhaled nitric oxide data were collected from each subject. A Meso Scale Discovery (MSD) electrochemiluminescence platform was applied to access serum levels of 33 cytokines. Serum cytokine profile was compared between mild and moderate-to-severe allergic asthmatics, and the correlation between serum cytokine levels, lung function and exhaled nitric oxide were analyzed.

Results: Moderate-to-severe allergic asthmatics displayed higher levels of eotaxin-1, eotaxin-2, MCP-1, MCP-2, MCP-3, YKL-40 and lower IL-23, IL-31 and TRAIL in serum in comparison with mild allergic asthmatics. Serum YKL-40, eotaxin-1 and MCP-1 had the best ability to discriminate mild and moderate-to-severe allergic asthmatics, with an AUC of 0.833, 0.811 and 0.760. Serum IP-10 was positively correlated with FeNO levels, while FnNO displayed a strong positive correlation with serum IL-25.

Conclusion: Compared with mild allergic asthmatics, significant increase in serum eotaxin-1, eotaxin-2, MCP-1, MCP-2, MCP-3, YKL-40 and decrease in serum IL-23, IL-31 and TRAIL was noted in moderate-to-severe allergic asthmatics. YKL-40, eotaxin-1 and MCP-1 might be candidate biomarkers in reflecting severity in allergic asthma patients.

Keywords: allergic asthma, cytokine, YKL-40, eotaxin, MCP

\section{Introduction}

Asthma is one of the most rapidly growing disorder among chronic respiratory diseases, affecting people from childhood to old age. Despite adequate medication administered under the guidance of Global Initiative for Asthma (GINA), 5-10\% of asthmatics remain only partially controlled or even uncontrolled and require further add-on therapy such as oral cortisone or novel biologic medication, and they suffer from increased mortality, increased healthcare costs and reduced quality of life. ${ }^{1}$ Severe asthma is, therefore, a nonnegligible burden for patients, their families, and the healthcare system.

Allergic asthma, defined as asthma associated with sensitization to environmental allergens, is the most common and most well-studied subtype of asthma, accounting for $60-80 \%$ of the whole asthma population. ${ }^{2,3}$ It has long been evident that allergic asthma is a prototypical Type 2 inflammation-driven disease, which is characterized based on release of type 2 cytokines (such as IL-4, IL-5 and IL-13) by T-helper cell type 2 (Th2) cells or innate lymphoid cells-type 2 (ILC2), followed by IgE class switching, eosinophil recruitment, and airway hyperresponsiveness (AHR). Thus, the majority of 
existing biological therapies are T2-targeted, like anti-IgE (omalizumab), anti-IL-5/IL-5R (mepolizumab, benralizumab) and anti-IL-4R (dupilumab). Unfortunately, although the clinical outcome of those biologics is favorable overall, some patients could not get a satisfactory response, indicating that solely targeting T2-related cytokine might not be enough. From previous studies we could come to the conclusion that other Th subsets, eosinophils, neutrophils, basophils, mast cells, macrophages also function at certain stage during its pathogenesis. ${ }^{4}$ Therefore, it is crucial to take them into consideration when it comes to optimize treatment strategy for allergic asthma.

Cytokines play a key role in the immunopathogenesis of asthma and are potent candidates in reflecting ongoing pathophysiological changes among patients and monitoring disease advancing. The cytokine network might have a difference in mild and moderate-to-severe allergic asthma patients and lead to different clinical outcome. In light of better understanding of the underlying mechanism, improving treatment regimens and decreasing disease burden, it is essential to investigate whether patients in different severity confer different cytokine expression. ${ }^{5}$

In the present study, we hypothesized that serum cytokine profile had a difference between mild and moderate-tosevere allergic asthmatics, and that these differences were associated with clinical heterogeneity. Thus, we quantified the level of 33 cytokines in serum samples from allergic asthma patients with different disease severity using Meso Scale Discovery (MSD) electrochemiluminescence platform, and analyzed the correlations between serum cytokine concentration, lung function and fractional exhaled nitric oxide levels.

\section{Materials and Methods}

\section{Subjects}

The study was approved by the ethic committee of Ruijin Hospital (No.2019-YK061) and was conducted in accordance with the Declaration of Helsinki. A total of 40 allergic asthma patients from outpatient clinic in Shanghai were enrolled in this study. Inclusion criteria included a diagnosis of asthma by physician according to the diagnosis criteria of the Global Strategy for Asthma Management and Prevention guidelines ${ }^{6}$ for at least one year, and a positive result of serum allergen-specific $\operatorname{IgE}(>0.35 \mathrm{kUA} / \mathrm{L})$ to at least one allergen tested and a total $\operatorname{IgE}>60 \mathrm{kU} / \mathrm{L}$. Exclusion criteria included poor medication compliance, history of cancer, autoimmune diseases and other respiratory or cardiovascular diseases. Patients were divided into two groups: those taking step 1 and step 2 medication and did not have any exacerbation in the past year were selected as mild group, while patients taking step 4 or step 5 medication routinely and had 2 or more exacerbations which needed urgent-care visits in the past year were categorized as moderate-to-severe group. To better display the difference between patients with different disease severity, patients taking step 3 medication were not included. At time of visit, all patients were in stable state of asthma, had no infection, no asthma exacerbation, no oral cortisone use or change in medication in the past month. Venous blood samples, ACT score and spirometry data was collected on all subjects according to ATS/European Respiratory Society (ERS) guidelines ${ }^{7}$ at the same day. All patients were informed about the purpose of the study and provided a written consent.

\section{Fractional Exhaled Nitric Oxide Measurement}

Fractional exhaled nitric oxide measurement was performed prior to spirometry test using Sunvou-CA2122 system (Sunvou, China) as previously described. ${ }^{8}$ Patients were required to avoid smoking, exercising and consuming nitraterich foods (such as sausage, broccoli, lettuce, celery and cabbage) one hour before testing. Fractional exhaled nitric oxide (FeNO) and nasal nitric oxide (FnNO) levels were measured under the guidance of ATS guidelines for online NO measurement in adults. ${ }^{9}$ Alveolar nitric oxide (CaNO) was accessed using a simplified estimation method based on fractional concentration of exhaled $\mathrm{NO}$ at 50 and $200 \mathrm{~mL} / \mathrm{s} .{ }^{10}$ Any exhalation that did not meet the ATS requirements was automatically rejected by the Sunvou system.

\section{Total IgE and Specific IgE Detection}

Serum total IgE and allergen-specific IgE to five common inhaled allergens in Shanghai (mold, dust mite, cat dander, dog dander and cockroach) were measured using the Phadia 250 fluorescence enzyme immunoassay system (Phadia, Sweden) according to the manufacturer's instructions. Serum allergen-specific IgE levels $>0.35 \mathrm{kUA} / \mathrm{L}$ were considered positive. 


\section{Meso Scale Discovery Assay}

Serum cytokine levels including Th1-related cytokines (IFN- $\gamma$, IP-10, TNF- $\beta$, IL-18), Th2-related cytokines (IL-4, IL-5, IL-6, IL-13, IL-17E, IL-31, IL-33, TARC, TSLP), Th9-related cytokine IL-9, Th17-related cytokines (IL-17A, IL-21, IL22, IL-23), Treg-related cytokine IL-10, monocyte-related cytokines (IL-27, IL-1 $\beta$, MCP-1, MCP-2, MCP-3, MCP-4), eosinophil-related cytokines (eotaxin-1, eotaxin-2, eotaxin-3, GM-CSF) and airway remodeling-related cytokines (VEGF-A, SDF-1 $\alpha$, YKL-40, TRAIL) were measured by MSD using U-PLEX Biomarker Group 1 (hu) Assays (MSD, USA) according to the instructions given by the manufacturer.

\section{Statistical Analysis}

Statistical analyses were performed in SPSS 25.0 software package (IBM Corp. Armonk, NY, USA) and GraphPad Prism 8.0 (GraphPad software, San Diego, CA, USA). Data was presented as mean \pm SD for quantitative data and as number and percentage for categorical data. Comparisons between different groups were made by independent samples $t$-test for quantitative statistics and chi-square test for categorical statistics. Receiver operating characteristic (ROC) curves were analyzed to identify candidate biomarkers to distinguish mild and moderate-to-severe allergic asthma patients and to provide their optimal cut-off values. Correlation analysis between quantitative variables was done using Spearman's correlations. A P-value $<0.05$ was considered as statistically significant.

\section{Results}

\section{Patient Characteristics}

The study consisted of 40 patients diagnosed with allergic asthma (mild, $n=22$; moderate-to-severe, $n=18$ ). Clinical characteristics of all subjects are summarized in Table 1. In general, patients in mild group were younger in those in moderate-to-severe group ( $(32.3 \pm 9.6)$ years vs $(44.0 \pm 15.0)$ years, $P=0.005)$, and their lung function (including $\mathrm{FVC} \%$, FEV1\%, FEV1/FVC\%, MEF75\%, MEF50\% but not MEF25\%) along with ACT score were significantly better (for FVC $\%$, FEV1\% and MEF75\%, $P<0.001$; for FEV1/FVC $\%$ and ACT score, $P<0.01$; for MEF50\%, $P=0.013$ ). Mold sensitization was more common in moderate-to-severe group $(P=0.025)$ while patients in mild group were mostly sensitized to dust mite. There was no significant difference regarding BMI, IgE, smoking status or gender distribution between two groups.

\section{Difference of Serum Cytokine Profile Between Mild and Moderate-to-Severe Allergic Asthma Patients}

We measured serum concentration of 33 cytokines in all subjects, including Th1-related cytokines (IFN- $\gamma$, IP-10, TNF- $\beta$, IL-18), Th2-related cytokines (IL-4, IL-5, IL-6, IL-13, IL-17E, IL-31, IL-33, TARC, TSLP), Th9-related cytokine IL-9, Th17-related cytokines (IL-17A, IL-21, IL-22, IL-23), Treg-related cytokine IL-10, monocyte-related cytokines (IL-27, IL$1 \beta$, MCP-1, MCP-2, MCP-3, MCP-4), eosinophil-related cytokines (eotaxin-1, eotaxin-2, eotaxin-3, GM-CSF) and airway remodeling-related cytokines (VEGF-A, SDF-1 $\alpha$, YKL-40, TRAIL) using Meso Scale Discovery method. The overall results are displayed in Table 2, and cytokines with significant difference between mild and moderate-to-severe group are demonstrated in Figure 1. In general, levels of Th2-related cytokines in serum were higher in moderate-to-severe allergic asthmatics, among which IL-31 was an exception $(P=0.004)$. Among Th17-related cytokines, a significant increase of serum IL-23 level was observed in mild allergic asthmatics $(P=0.008)$. Serum eotaxin-1, eotaxin-2, MCP-1, MCP-2, MCP3 , YKL-40 levels were significantly higher in moderate-to-severe group $(P=0.001,0.017,0.003,0.018,0.036,0.022)$, while serum TRAIL concentration was significantly lower than that in mild group $(P=0.045)$.

For the 9 cytokines that differ significantly among two groups, a correlation matrix between their levels and all 33 cytokines tested is displayed in Figure 2. Besides correlated with each other $(P<0.001)$, IL-23 and IL-31 was also positively correlated with IL-17A, IL-25 (all $P<0.001$ ), GM-CSF, TSLP, IL-21, IL-33 (all $P<0.01$ ), IL-9 (all $P<0.05$ ), and negatively correlated with eotaxin-2 $(P<0.01)$, eotaxin-1 (IL-23, $P<0.05$; IL-31, $P<0.01)$ and YKL-40 $(P<0.05)$. MCP-1 displayed a positive correlation with IL-13, VEGF-A, eotaxin-1, eotaxin-3, TARC, TNF- $\beta$, MCP-2, MCP-3, MCP-4 (all $P<0.001$ ), IL-18, eotaxin-2, YKL-40 (all $P<0.01$ ), IL-4, IL-5 (all $P<0.05$ ). MCP-2 was correlated positively 
Table I Baseline Characteristics of Patients with Mild and Moderate-to-Severe Allergic Asthma

\begin{tabular}{|l|l|l|l|}
\hline & Mild & Moderate-to-Severe & $P$ value \\
\hline N & 22 & 18 & \\
Male: Female & $9: 13$ & $7: 11$ & 0.897 \\
Age, years & $32.3 \pm 9.6$ & $44.0 \pm 15.0$ & $0.005^{* *}$ \\
BMI, kg/m & $23.2 \pm 3.3$ & $23.4 \pm 4.5$ & 0.849 \\
Smoking status, current: past: never & $0: 1: 21$ & $0.1: 17$ & 0.884 \\
FVC, \%predicted & $98.77 \pm 10.36$ & $78.03 \pm 18.44$ & $<0.00 I^{* * *}$ \\
FEVI, \%predicted & $95.36 \pm 12.53$ & $63.64 \pm 25.06$ & $<0.00 I^{* * *}$ \\
FEVI/FVC, \%predicted & $96.91 \pm 11.52$ & $79.20 \pm 22.75$ & $0.007^{* *}$ \\
MEF75, \%predicted & $91.49 \pm 19.44$ & $51.18 \pm 33.13$ & $<0.00 I^{* * *}$ \\
MEF50, \%predicted & $79.55 \pm 19.67$ & $49.06 \pm 40.87$ & $0.013^{*}$ \\
MEF25, \%predicted & $70.55 \pm 28.06$ & $46.05 \pm 43.35$ & 0.078 \\
ACT score & $22.72 \pm 1.81$ & $17.53 \pm 4.45$ & $0.002^{* *}$ \\
IgE, kU/L & $761.9 \pm 1164.7$ & $473.2 \pm 834.7$ & 0.501 \\
Allergen, positive result (number, \%) & & & \\
Mold & $2,9.1 \%$ & $7,38.9 \%$ & $0.025^{*}$ \\
Dust mite & $21,95.4 \%$ & $11,61.1 \%$ & $0.007^{* *}$ \\
Cat dander & $7,31.8 \%$ & $2,11.1 \%$ & 0.119 \\
Dog dander & $7,31.8 \%$ & $4,22.2 \%$ & 0.499 \\
Cockroach & $5,22.7 \%$ & $1,5.6 \%$ & 0.130 \\
\hline
\end{tabular}

Notes: Data are presented as mean \pm standard deviation or number, percentage. $* P<0.05 ; * * P<0.01 ; * * * P<0.001$.

Abbreviations: BMI, body mass index; FVC, forced vital capacity; FEVI, forced expiratory volume in the first second; MEF, maximal expiratory flow; ACT, asthma control test; IgE, immunoglobulin E.

with IL-13, eotaxin-1, MCP-1, MCP-4, TARC, TNF- $\beta$, VEGF-A (all $P<0.001$ ), IL-18 (all $P<0.01$ ), IL-5, eotaxin-2, eotaxin-3, MCP-3, IP-10 (all $P<0.05$ ). YKL-40 showed a negative correlation with IL-17A, IL-21, TRAIL (all $P<0.01$ ), IL-23, IL-25, IL-31 (all $P<0.05$ ), and a positive correlation with eotaxin-1 $(P<0.001)$, eotaxin-2, VEGF-A, MCP-1, MCP-3 (all $P<0.01$ ).

To find a candidate serum biomarker in distinguishing mild and moderate-to-severe allergic asthmatics, ROC analyses were performed, and main results are summarized in Table 3 and Figure 3. The area under the curves (AUCs) of YKL40, eotaxin-1, MCP-1, IL-23, IL-31, eotaxin-2, MCP-2, MCP-3 were $0.833,0.811,0.760,0.754,0.749,0.725,0.720$ and 0.707 , respectively. The best cut-off points obtained from ROC curve analysis with the greatest likelihood ratios for YKL-40, eotaxin-1, MCP-1, IL-23, IL-31, eotaxin-2, MCP-2, MCP-3 were 33,753, 134.1, 142.9, 1.035, 11.78, 1210 , 43.06 and $5.847 \mathrm{pg} / \mathrm{mL}$, respectively. TRAIL was excluded from the result as it did not reach an AUC of 0.7.

\section{Correlations Between Serum Cytokine Levels and Lung Function}

We further explored the relationship between serum cytokine levels and lung function of allergic asthmatics. Main results were shown in Table 4, and a correlation matrix was displayed in Figure 4. Serum eotaxin-1 level was negatively correlated with FEV1\%, FVC\%, FEV1/FVC\%, MEF75\%, MEF50\% and MEF25\% $(P=0.003,0.003,0.012,0.006,0.013$, 0.017 , respectively). Eotaxin-2 was negatively correlated with FEV1\%, FEV1/FVC\%, MEF75\%, MEF50\%, MEF $25 \%$ $(P=0.010,0.007,0.008,0.003,0.006$, respectively). YKL-40 displayed a negative correlation with $\mathrm{FEV} 1 \%, \mathrm{FVC} \%$, MEF75\%, MEF50\% and MEF25\% ( $P=0.003,0.001,0.016,0.022,0.026$, respectively). Serum TARC concentration was inversely correlated with FEV1\% $(P=0.023)$ and $\mathrm{FVC} \%(P=0.019)$. Among the cytokines tested, IL-21 was the only one positively correlated with FEV1\% $(P=0.049)$. Serum IL-23 and IL-31 was positively correlated with FEV1/FVC\% $(P=0.015,0.034$, respectively).

\section{Correlations Between Serum Cytokine Levels and FeNO, CaNO and FnNO Levels}

We next analyzed correlations between FeNO, $\mathrm{CaNO}$ and FnNO levels and serum cytokine levels. Main results are summarized in Table 5, and a correlation matrix between cytokine levels and FeNO, CaNO and FnNO levels is 
Table 2 Serum Cytokine Levels in Mild and Moderate-to-Severe Allergic Asthma Patients

\begin{tabular}{|c|c|c|c|}
\hline & Mild (pg/mL) & Moderate-to-Severe $(\mathrm{pg} / \mathrm{mL})$ & $P$ value \\
\hline \multicolumn{4}{|c|}{ ThI-related cytokines } \\
\hline IFN- $\gamma$ & $15.0327 \pm 18.5494$ & $9.908 I \pm 5.4423$ & 0.265 \\
\hline IP-10 & $219.5332 \pm 81.9197$ & $179.8225 \pm 67.2624$ & 0.107 \\
\hline TNF- $\beta$ & $1.3760 \pm 1.1251$ & $1.6338 \pm 0.6957$ & 0.402 \\
\hline IL-18 & $382.3738 \pm 180.2698$ & $437.5303 \pm 237.5416$ & 0.409 \\
\hline \multicolumn{4}{|c|}{ Th2-related cytokines } \\
\hline IL-4 & $0.0552 \pm 0.1760$ & $0.0715 \pm 0.0508$ & 0.168 \\
\hline IL-5 & $0.8976 \pm 0.446 \mathrm{I}$ & $1.0189 \pm 0.6217$ & 0.477 \\
\hline IL-6 & $1.9240 \pm 3.6287$ & $10.6227 \pm 37.7049$ & 0.343 \\
\hline IL-13 & $2.9740 \pm 1.4682$ & $3.6162 \pm 0.9953$ & 0.115 \\
\hline IL-I7E & $1.4924 \pm 0.8692$ & $1.2052 \pm 0.7952$ & 0.300 \\
\hline IL-3I** & $|3.337| \pm 6.2233$ & $7.9754 \pm 3.7165$ & $0.004 * *$ \\
\hline IL-33 & $0.8603 \pm 0.5922$ & $0.5982 \pm 0.2588$ & 0.071 \\
\hline TARC & $179.4743 \pm 325.5475$ & $248.3678 \pm 154.6949$ & 0.415 \\
\hline TSLP & $1.4226 \pm 0.5462$ & $1.388 \mathrm{I} \pm 0.4468$ & 0.831 \\
\hline \multicolumn{4}{|c|}{ Th9-related cytokine } \\
\hline IL-9 & $0.5086 \pm 0.5584$ & $0.3077 \pm 0.2093$ & 0.157 \\
\hline \multicolumn{4}{|c|}{ Th I7-related cytokines } \\
\hline IL-I7A & $3.8704 \pm 2.6369$ & $3.0720 \pm 1.6177$ & 0.269 \\
\hline IL-2I & $67.1995 \pm 146.7237$ & $3.1749 \pm 1.8464$ & 0.053 \\
\hline IL-22 & $0.7479 \pm 0.9150$ & $0.6558 \pm 0.3282$ & 0.687 \\
\hline IL-23** & $2.3996 \pm 1.7093$ & $0.9643 \pm 0.9247$ & $0.008 * *$ \\
\hline \multicolumn{4}{|c|}{ Treg-related cytokine } \\
\hline IL-10 & $0.4019 \pm 0.1935$ & $0.4314 \pm 0.1231$ & 0.579 \\
\hline \multicolumn{4}{|c|}{ Monocyte-related cytokines } \\
\hline IL-27 & $650.1278 \pm 315.4457$ & $759.9686 \pm 469.0485$ & 0.383 \\
\hline IL-I $\beta$ & $3.9250 \pm 16.9320$ & $4.6160 \pm 18.2887$ & 0.902 \\
\hline MCP-I** & $134.0964 \pm 59.9890$ & $206.7393 \pm 83.5712$ & $0.003 * *$ \\
\hline MCP-2* & $38.4753 \pm 13.8157$ & $49.2572 \pm 13.6278$ & $0.018^{*}$ \\
\hline MCP-3* & $4.7400 \pm 1.7576$ & $6.7599 \pm 3.9217$ & $0.036 *$ \\
\hline MCP-4 & $99.7898 \pm 109.3274$ & $133.5642 \pm 70.0432$ & 0.264 \\
\hline \multicolumn{4}{|c|}{ Eosinophil-related cytokines } \\
\hline Eotaxin-I*** & $101.8066 \pm 149.2978$ & $191.8802 \pm 83.5456$ & $<0.00 I^{* * *}$ \\
\hline Eotaxin-2* & $1203.4756 \pm 90 \mid .7847$ & $1938.4275 \pm 936.9545$ & $0.017^{*}$ \\
\hline Eotaxin-3 & $84.7753 \pm 133.9955$ & $37.7562 \pm 20.2448$ & 0.119 \\
\hline GM-CSF & $0.0733 \pm 0.0430$ & $0.0911 \pm 0.0837$ & 0.391 \\
\hline \multicolumn{4}{|c|}{ Airway remodeling-related cytokines } \\
\hline VEGF-A & $74.0250 \pm 74.6739$ & $111.3350 \pm 61.3555$ & 0.097 \\
\hline SDF-I $\alpha$ & $842.2624 \pm 263.7370$ & $893.5355 \pm 255.9779$ & 0.539 \\
\hline YKL-40* & $23,210.1859 \pm 15,116.1899$ & $51,974.8527 \pm 54,283.5695$ & $0.022^{*}$ \\
\hline TRAIL* & $134.3080 \pm 37.1827$ & $110.7160 \pm 34.0288$ & $0.045^{*}$ \\
\hline
\end{tabular}

Notes: Data are presented as mean \pm standard deviation. $* P<0.05 ; * * P<0.01 ; * * * p<0.001$.

Abbreviations: Th, T helper cell; IFN- $\gamma$, interferon- $\gamma$; IP-I0, interferon- $\gamma$-induced protein I0; TNF- $\beta$, tumor necrosis factor- $\beta$; IL, interleukin; TARC, thymus and activationregulated chemokine; TSLP, thymic stromal lymphopoietin; MCP, monocyte chemoattract protein; GM-CSF, granulocyte-macrophage colony-stimulating factor; VEGF-A, vascular endothelial growth factor-A; SDF-I $\alpha$, stromal derived factor-I $\alpha$; TRAIL, tumor necrosis factor-related apoptosis-inducing ligand.

displayed in Figure 5. FeNO showed a positive correlation with serum IP-10 $(P=0.042)$ while CaNO levels were positively correlated with IL-4 and IL-1 $\beta(P=0.031,0.018$, respectively). FnNO levels were positively correlated with serum IL-4, IL-25, IL-31, IL-9, GM-CSF and negatively correlated with serum eotaxin-2 ( $P=0.042,0.009$, $0.047,0.030,0.037,0.040$, respectively). 
A

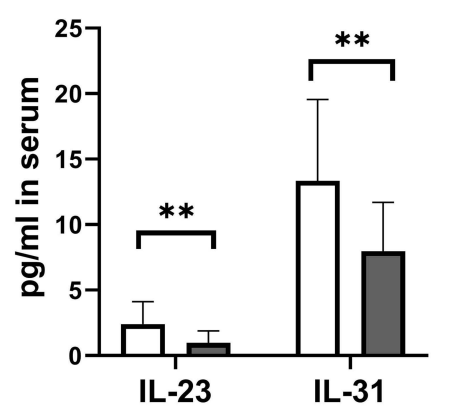

C

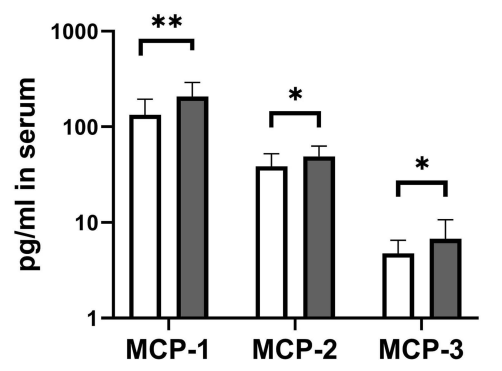

B

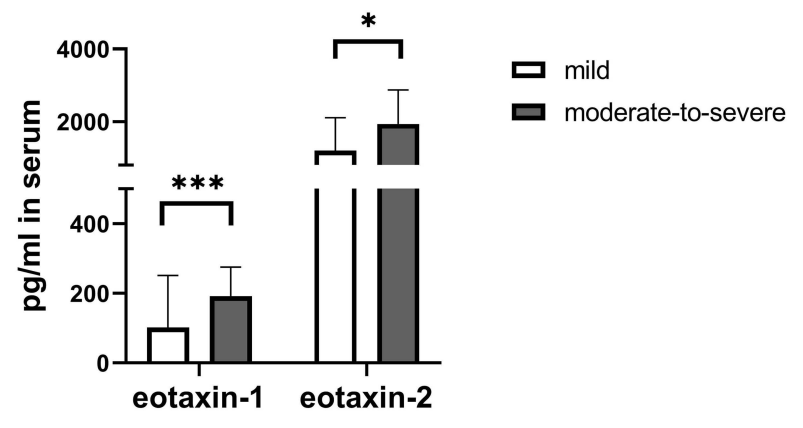

D

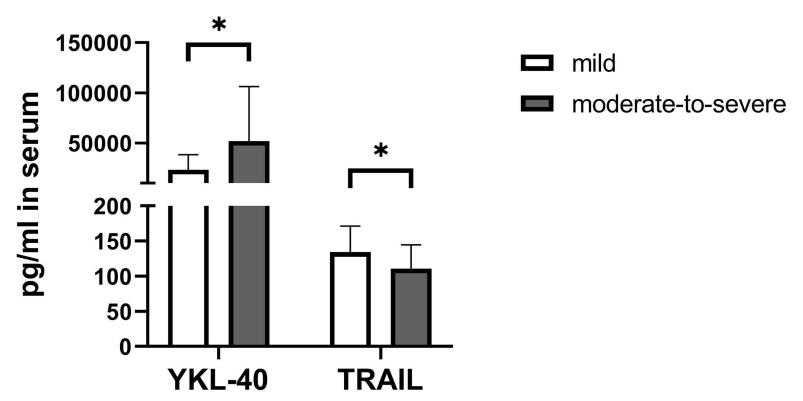

Figure I Serum cytokine concentrations of the most discriminative biomarkers (A) IL-23 and IL-3I, (B) eotaxin-I and eotaxin-2, (C) MCP-I, MCP-2 and MCP-3, (D) YKL40 and TRAIL in mild and moderate-to-severe allergic asthmatics. $* P<0.05 ; * * P<0.01 ; * * * P<0.001$.

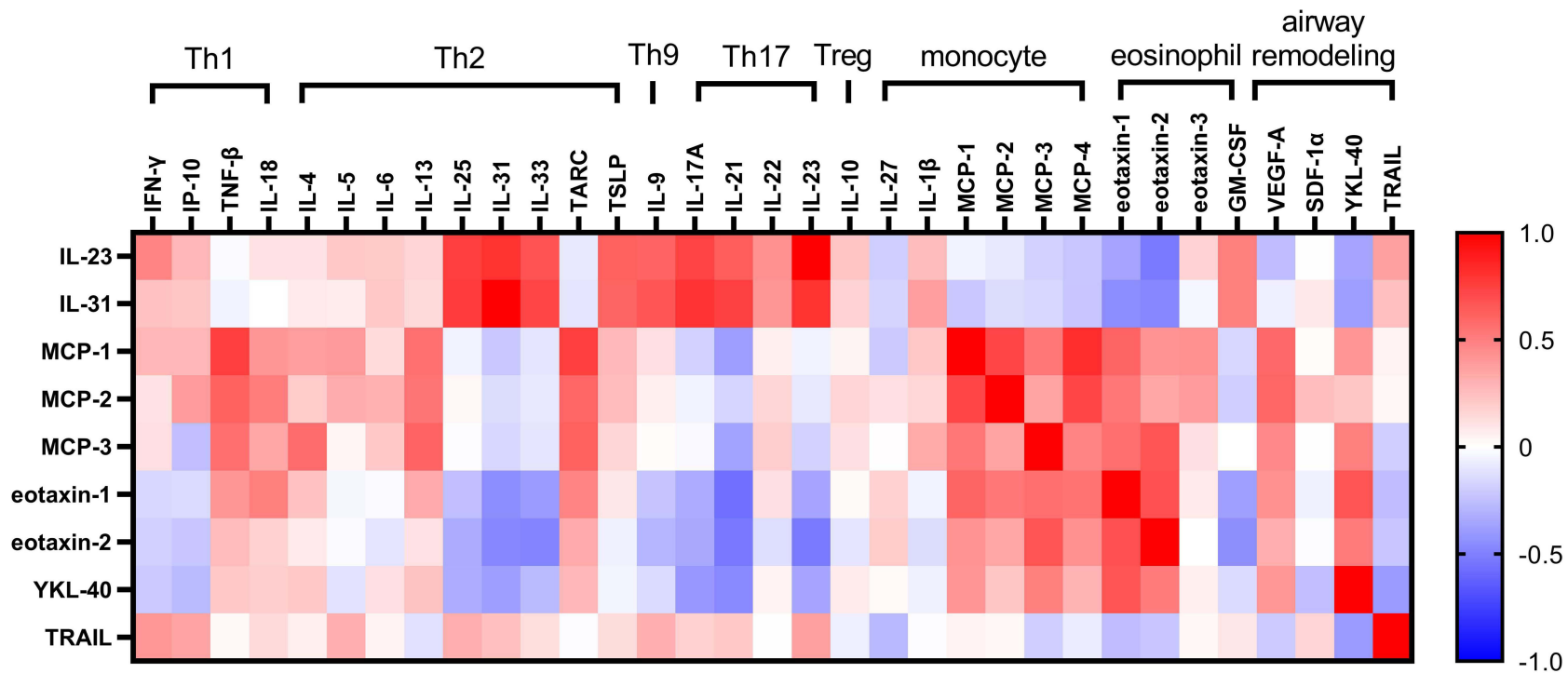

Figure 2 Correlation matrix for 9 discriminative biomarkers with all 33 cytokines tested. Blue represented a negative correlation index while red represented a positive one. The darker the color, the higher the correlation index was.

\section{Discussion}

Allergic asthma is a heterogeneous disorder with complex immunopathology. In the present study, we portrayed differences of cytokine profile between serum samples of mild and moderate-to-severe allergic asthmatics and analyzed 
Table 3 The AUC, Cut-off, Sensitivity and Specificity Values for Mild and Moderate-to-Severe Allergic Asthma Patients

\begin{tabular}{|l|l|l|l|l|l|}
\hline & AUC & P value & Cut-off $(\mathbf{p g} / \mathbf{m L})$ & Sensitivity $(\mathbf{9 5 \%} \mathbf{~ C l})$ & Specificity $(\mathbf{9 5 \%} \mathbf{C l})$ \\
\hline YKL-40 & 0.833 & $<0.00 I^{* * *}$ & 33,753 & $72.22(49.13-87.50)$ & $90.91(72.19-98.38)$ \\
Eotaxin-I & $0.8 I I$ & $<0.00 I^{* * *}$ & 134.1 & $72.22(49.13-87.50)$ & $72.73(51.85-86.85)$ \\
MCP-I & 0.760 & $0.005^{* *}$ & 142.9 & $77.78(54.79-91.00)$ & $72.73(51.85-86.85)$ \\
IL-23 & 0.754 & $0.015^{*}$ & 1.035 & $71.43(45.35-88.28)$ & $83.33(60.78-94.16)$ \\
IL-3I & 0.749 & $0.014^{*}$ & 11.78 & $86.67(62.12-97.63)$ & $57.89(36.28-76.86)$ \\
Eotaxin-2 & 0.725 & $0.016^{*}$ & 1210 & $83.33(60.78-94.16)$ & $54.55(34.66-73.08)$ \\
MCP-2 & 0.720 & $0.018^{*}$ & 43.06 & $66.67(43.75-83.72)$ & $72.73(51.85-86.85)$ \\
MCP-3 & 0.707 & $0.026^{*}$ & 5.847 & $61.11(38.62-79.69)$ & $72.73(51.85-86.85)$ \\
\hline
\end{tabular}

Notes: $* P<0.05 ; * * P<0.01 ; * * * P<0.001$.

Abbreviations: AUC, area under curve; $\mathrm{Cl}$, confidence interval.

the correlations between cytokine concentration, lung function parameters as well as exhaled nitric oxidate levels. Previous studies have examined the difference of cytokine profile in bronchial biopsy samples or bronchoalveolar lavage. ${ }^{11,12}$ Indeed, those samples could best reflect the condition in airways. Nevertheless, serum sample would be more practical in clinical settings, making it a feasible alternative. To the best of our knowledge, this is the first research evaluating all those 33 cytokines simultaneously in serum samples of allergic asthma patients and analyzed their correlation with both lung function parameters and exhaled nitric oxide levels.

The present study elucidated that moderate-to-severe allergic asthmatics expressed higher levels of eotaxin-1, eotaxin-2, MCP-1, MCP-2, MCP-3, YKL-40 and lower IL-23, IL-31 and TRAIL in serum in comparison with mild allergic asthmatics. Serum eotaxin-1, eotaxin-2, YKL-40 levels were negatively correlated with lung function parameters for both main and small airways. YKL-40, eotaxin-1 and MCP-1 had the best ability to discriminate mild and moderateto-severe allergic asthmatics, with an AUC of $0.833,0.811$ and 0.760 . The concentration of serum IP-10 displayed a significantly positive correlation with FeNO levels, while CaNO levels were positively correlated with serum IL-4 and IL-1 $\beta$. FnNO levels were positively correlated with serum IL-4, IL-25, IL-31, IL-9, GM-CSF and negatively correlated with serum eotaxin-2.

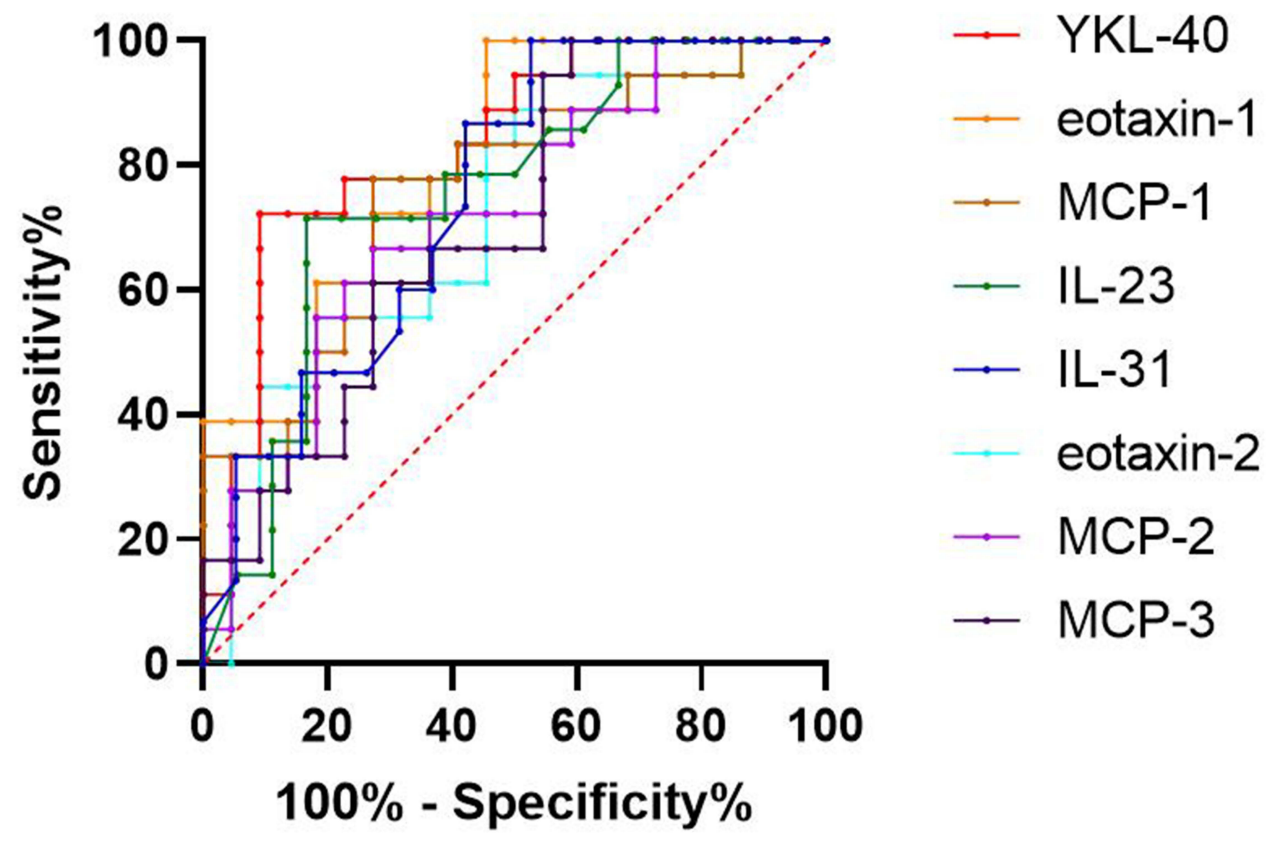

Figure 3 Receiver operating characteristic (ROC) curves of YKL-40, eotaxin-I, MCP-I, IL-23, IL-3I, eotaxin-2, MCP-2 and MCP-3 for discriminating mild and moderate-tosevere allergic asthmatics. 
Table 4 Correlations Between Cytokine Levels and Lung Function Parameters

\begin{tabular}{|c|c|c|c|}
\hline Parameter & Cytokine & Correlation Coefficient & $P$ value \\
\hline \multirow[t]{6}{*}{ FEVI\% } & YKL-40 & -0.478 & $0.003^{* *}$ \\
\hline & Eotaxin-I & -0.472 & $0.003^{* *}$ \\
\hline & Eotaxin-2 & -0.418 & $0.010 *$ \\
\hline & TARC & -0.372 & $0.023 *$ \\
\hline & MCP-3 & -0.372 & $0.023^{*}$ \\
\hline & $|\mathrm{IL}-2|$ & 0.326 & $0.049 *$ \\
\hline \multirow[t]{5}{*}{ FVC\% } & YKL-40 & -0.506 & $0.00 I^{* *}$ \\
\hline & Eotaxin-I & -0.477 & $0.003^{* *}$ \\
\hline & IL-22 & -0.428 & $0.008^{* *}$ \\
\hline & MCP-3 & -0.423 & $0.009 * *$ \\
\hline & TARC & -0.385 & $0.019 *$ \\
\hline \multirow[t]{4}{*}{ FEVI/FVC } & Eotaxin-2 & -0.434 & $0.007^{* *}$ \\
\hline & Eotaxin-I & -0.411 & $0.012^{* *}$ \\
\hline & IL-23 & 0.397 & $0.015^{*}$ \\
\hline & $|\mathrm{IL}-3|$ & 0.350 & $0.034 *$ \\
\hline \multirow[t]{3}{*}{ MEF75\% } & Eotaxin-I & -0.440 & $0.006^{* *}$ \\
\hline & Eotaxin-2 & -0.432 & $0.008^{* *}$ \\
\hline & YKL-40 & -0.394 & $0.016 *$ \\
\hline \multirow[t]{4}{*}{ MEF $50 \%$} & Eotaxin-2 & -0.479 & $0.003^{* *}$ \\
\hline & Eotaxin-I & -0.404 & $0.013^{*}$ \\
\hline & YKL-40 & -0.376 & $0.022 *$ \\
\hline & IL-23 & 0.329 & $0.047^{*}$ \\
\hline \multirow[t]{3}{*}{ MEF $25 \%$} & Eotaxin-2 & -0.443 & $0.006^{* *}$ \\
\hline & Eotaxin-I & -0.390 & $0.017^{*}$ \\
\hline & YKL-40 & -0.366 & $0.026^{*}$ \\
\hline
\end{tabular}

Notes: Correlations between serum cytokine levels and FEVI\%, FVC\%, FEVI/FVC\%, MEF75\%, MEF50\% and MEF25\% were analyzed. All correlations listed above were evaluated using Spearman correlation coefficient analysis. $* P<0.05 ; * * P<0.01$.

Abbreviations: FEVI, forced expiratory volume in the first second; FVC, forced vital capacity; MEF, maximal expiratory flow.

The monocyte chemotactic proteins (MCPs) are considered as a subfamily of the CC chemokines, which play an important role in allergic responses via induction of mast cell activation and release of leukotriene $\mathrm{C} 4$ into the airway, resulting in airway hyperresponsiveness. ${ }^{13}$ Enhanced expression of MCP-1 was observed in bronchial tissue and BAL fluids of asthmatic subjects by previous study. ${ }^{14}$ In this study, we discovered that MCP-1, MCP-2, MCP-3 concentration is also elevated in serum of moderate-to-severe allergic asthmatics than that of mild allergic asthmatics. Furthermore,

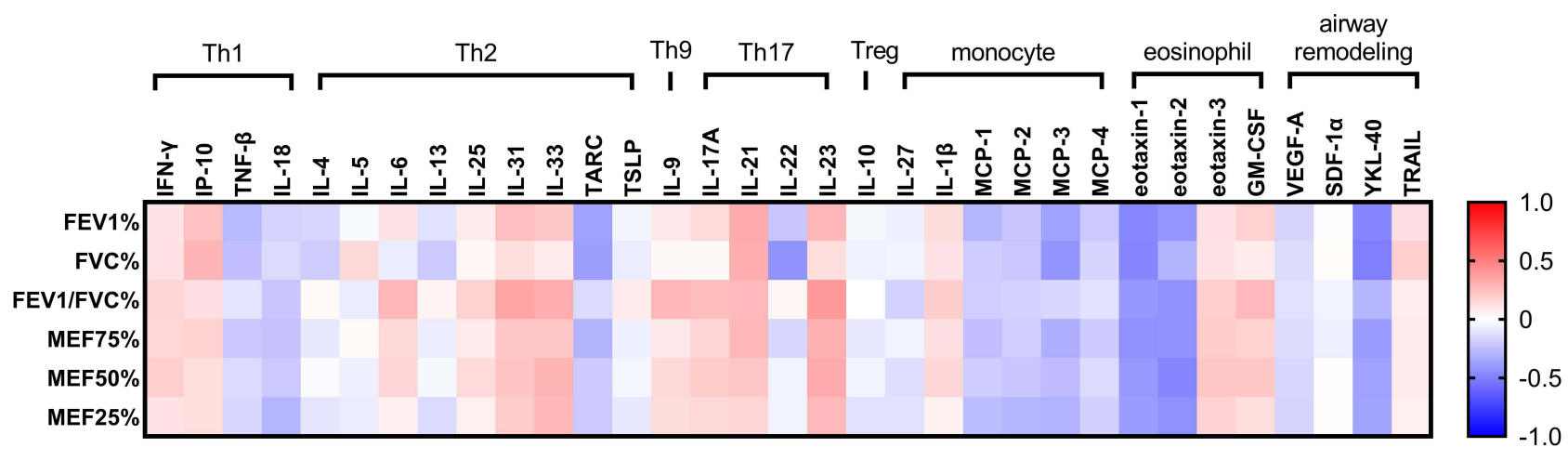

Figure 4 Correlation matrix for lung function parameters with all 33 cytokines tested. Blue represented a negative correlation index while red represented a positive one. The darker the color, the higher the correlation index was. 
Table 5 Correlations Between Serum Cytokine Levels and Exhaled Nitric Oxide Levels

\begin{tabular}{|l|l|l|l|}
\hline Parameter & Cytokine & Correlation Coefficient & P value \\
\hline FeNO & IP-IO & 0.417 & $0.042^{*}$ \\
CaNO & IL-I $\beta$ & 0.487 & $0.018^{*}$ \\
& IL-4 & 0.450 & $0.03 I^{*}$ \\
& IL-25 & 0.566 & $0.009^{*}$ \\
& IL-9 & 0.486 & $0.030^{*}$ \\
& GM-CSF & 0.469 & $0.037^{*}$ \\
& Eotaxin-2 & -0.463 & $0.040^{*}$ \\
& IL-4 & 0.459 & $0.042^{*}$ \\
& IL-3I & 0.449 & $0.047^{*}$ \\
\hline
\end{tabular}

Notes: Correlations between serum cytokine levels and FeNO, $\mathrm{CaNO}$ and FnNO were analyzed. All correlations listed above were evaluated using Spearman correlation coefficient analysis. $* P<0.05 ; * * P<0.01$.

Abbreviations: FeNO, fractional exhaled nitric oxide; $\mathrm{CaNO}$, alveolar nitric oxide; FnNO, nasal nitric oxide.

serum MCP-3 was negatively correlated with FEV1\% and FVC\%. Taken together, serum MCP levels are promising candidates in distinguishing mild and severe asthmatics.

Members in eotaxin family, including eotaxin-1 (CCL11), eotaxin-2 (CCL24) and eotaxin-3 (CCL26), are potent eosinophil chemoattractant promoting eosinophils migration to sites of inflammation. ${ }^{15}$ Previous study elucidated that all those three members were elevated in BAL fluids of asthmatics, and were associated with a decreased FEV1\%. ${ }^{16,17}$ Our study further discovered a significant elevation in both serum eotaxin-1 and eotaxin- 2 in moderate-to-severe allergic asthmatics compared with mild group. Eotaxin-1 and eotaxin-2 displayed a negatively correlation not only with FEV1\% but also with FEV1/FVC\%, MEF75\%, MEF50\% and MEF25\%, suggesting their role in both small and main airway obstruction. All these findings strongly indicates that eotaxin-1 and eotaxin-2 are related with an impaired lung function and are likely to be responsible for development of severe allergic asthma.

YKL-40, also named chitinase-3-like-1 (CHI3L1), is a chitinase-like glycoprotein related closely to airway remodeling. Previous study had shown that serum YKL-40 was elevated in asthma patients compared with healthy controls, ${ }^{18}$ additionally, elevated serum YKL-40 levels were associated with irreversible airway obstruction and severe exacerbations. ${ }^{19-21}$ YKL-40 is also elevated in COPD patients than Asthma-COPD Overlap patients, which may suggest its potential in distinguishing ACO patients from COPD patients. ${ }^{22}$ In this study, we found the concentration of YKL-40 in serum of mild-to-moderate allergic asthmatics to be higher than that of mild allergic asthmatics, and was negatively correlated with FEV1\%, MEF75\%, MEF50\% and MEF25\%, indicating that patients with high serum YKL-40 might have a tendency to progress into ACO or COPD, which calls for an urgent need to take step in prevention.

IL-23 has been identified as a novel member of IL-12 family. According to previous study, IL-23-Th17 cell axis is involved not only in causing antigen-induced neutrophil recruitment into the airways but also in the enhancement of Th2 cell-mediated eosinophil recruitment, indicating a role in airway inflammation formation. ${ }^{23,24}$ However, some other studies revealed that IL-23 performed a protective role in airway inflammation, suggesting that IL-23 may exert different

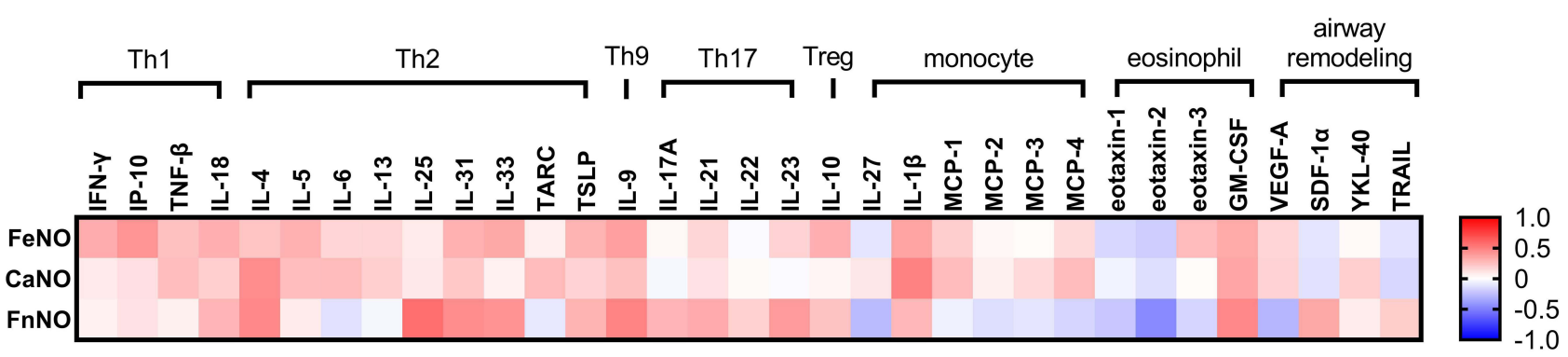

Figure 5 Correlation matrix for FeNO, CaNO and FnNO levels with all 33 cytokines tested. Blue represented a negative correlation index while red represented a positive one. The darker the color, the higher the correlation index was. 
function under different circumstances. ${ }^{25,26}$ IL-31 is a novel T helper type 2 effector cytokine participating in allergic inflammation in the lung, gut and skin. ${ }^{27}$ Although initial studies have found that IL-31 was elevated in serum of asthmatics in comparison with healthy controls, several animal studies suggest that it may reduce allergen-induced lung inflammation or play dual roles. ${ }^{28-30}$ In our study, we found that serum IL-23 and IL-31 levels to be higher in mild allergic asthma patients that in moderate-to-severe ones, additionally, they were both positively correlated with FEV1/ FVC\%. Moreover, both serum IL-23 and IL-31 levels were positively correlated with alarmins IL-25, IL-33 and TSLP, which were indicated to be among the first events in initiating allergic inflammation. ${ }^{31}$ Taken together, these findings suggest that IL-23 and IL-31 might play a role in the early phase of pathogenesis for allergic asthma.

Tumor necrosis factor-related apoptosis-inducing ligand (TRAIL) is a member of TNF family which has an important role in allergic airways inflammation and contributes to airway remodeling. ${ }^{32,33}$ It is expressed in the airway by various type of cells, including fibroblasts, epithelial, endothelial, smooth muscle cells and eosinophils. ${ }^{34}$ Our study demonstrated that moderate-to-severe allergic asthmatics expressed lower TRAIL than mild patients but was not correlated with lung function or NO levels. Moreover, TRAIL had poor ability in discriminating mild and moderate-to-severe allergic asthmatics in the ROC analysis. Therefore, more studies were needed to conclude the role of TRAIL in progressing disease severity.

IL-25 belongs to alarmin family, which is increased during allergen stimulation, and in turn enhance Th2-type airway inflammation. ${ }^{35-37}$ It was also discovered that high nasal IL-25 mRNA expression was associated with higher Th2 response. ${ }^{38}$ In this study, we observed a strong and significant association between FnNO levels and serum IL-25 levels. In combination with previous studies and our findings, we speculate that IL-25 might be one of the starters of T2-type inflammation in upper airways.

The main limitation of this study is a relatively small size. Results need to be validated in a larger cohort. Secondly, as this is a cross-sectional study, cause and effect between cytokine levels and disease severity could not be precisely concluded. Longitudinal follow-up studies will be needed to determine how these cytokines change through the course of disease and whether they lead to different prognosis. Furthermore, we only focused on serum cytokine levels, without accessing certain cell counts in blood. Nonetheless, our findings provide an integrative insight of difference in serum cytokine profile as well as their correlations with lung function and exhaled nitric oxide data in mild and moderate-tosevere asthmatics.

\section{Conclusion}

In this study, we demonstrated significant differences in serum cytokine profile between mild and moderate-to-severe allergic asthmatics. Moderate-to-severe allergic asthmatics presented higher serum levels of MCP-1, MCP-2, MCP-3, eotaxin-1, eotaxin-2 and YKL-40 and lower serum levels of IL-23, IL-31 and TRAIL in comparison with mild allergic asthmatics. In ROC analysis, YKL-40, eotaxin-1 and MCP-1 was potent in distinguishing allergic asthmatics with different severity. Our findings highlight the potential value of these cytokines in disease monitoring and might help mechanism studying as well as targeting therapeutic agents.

\section{Acknowledgments}

The present study was supported by grants from the Chinese National Natural Science Foundation (No.81870021); National Key research and development program (2016YFC0901103); Shanghai Municipal Key Clinical Specialty (shslczdzk02202). Lin Sun and Bo Peng are co-first authors for this study.

\section{Disclosure}

The authors report no conflicts of interest in this work.

\section{References}

1. Khurana S, Jarjour NN. Systematic approach to asthma of varying severity. Clin Chest Med. 2019;40(1):59-70. doi:10.1016/j.ccm.2018.10.004

2. Nanda A, Wasan AN. Asthma in Adults. Med Clin North Am. 2020;104(1):95-108. doi:10.1016/j.mcna.2019.08.013

3. Rehman A, Amin F, Sadeeqa S. Prevalence of asthma and its management: a review. JPMA. 2018;68(12):1823-1827. 
4. Zissler U, Esser-von Bieren J, Jakwerth CA, et al. Current and future biomarkers in allergic asthma. Allergy. 2016;71(4):475-494. doi:10.1111/ all. 12828

5. Agache I, Akdis C, Jutel M, et al. Untangling asthma phenotypes and endotypes. Allergy. 2012;67(7):835-846. doi:10.1111/j.13989995.2012.02832.x

6. Poga B, Mma C. The 2019 global initiative for asthma report: evidence-based or evidence-biased medicine? Therapies. 2021;76(1):57-58. doi:10.1016/j.therap.2020.02.021

7. Brusasco V, Viegi G. The ATS/ERS consensus on clinical Pulmonary function testing. Breathe. 2005;2(1):9-10.

8. Lei W, Li F, Tang X-M, et al. The comparison of two exhaled nitric oxide analyzers: NIOX VERO and SUNVOU-CA2122. J Breath Res. 2021;15 (2):026007. doi:10.1088/1752-7163/abd193

9. American Thoracic Society. European Respiratory Society. ATS/ERS recommendations for standardized procedures for the online and offline measurement of exhaled lower respiratory nitric oxide and nasal nitric oxide. Am J Respir Crit Care Med. 2005;171:912-930

10. Fan X, Zhao N, Yu Z, et al. Clinical utility of central and peripheral airway nitric oxide in aging patients with stable and acute exacerbated chronic obstructive pulmonary disease. Int J Gen Med. 2021;14:571-580. doi:10.2147/IJGM.S284688

11. Hosoki K, Ying S, Corrigan C, et al. Analysis of a panel of 48 cytokines in bal fluids specifically identifies IL-8 levels as the only cytokine that distinguishes controlled asthma from uncontrolled asthma, and correlates inversely with FEV1. PLoS One. 2015;10(5):e0126035. doi:10.1371/ journal.pone.0126035

12. Shannon J, Ernst P, Yamauchi Y, et al. Differences in airway cytokine profile in severe asthma compared to moderate asthma. Chest. $2008 ; 133$ (2):420-426. doi:10.1378/chest.07-1881

13. Van Coillie E, Van Damme J, Opdenakker G. The MCP/eotaxin subfamily of CC chemokines. Cytokine Growth Factor Rev. 1999;10(1):61.

14. Sousa AR, Lane SJ, Nakhosteen JA, et al. Increased expression of the monocyte chemoattractant protein-1 in bronchial tissue from asthmatic subjects. Am J Respir Cell Mol Biol. 1994;10(2):142. doi:10.1165/ajrcmb.10.2.8110469

15. Huber AK, Giles DA, Segal BM, et al. An emerging role for eotaxins in neurodegenerative disease. Clin Immunol. 2018;189:29-33. doi:10.1016/j. clim.2016.09.010

16. Coleman JM, Naik C, Holguin F, et al. Epithelial eotaxin-2 and eotaxin-3 expression: relation to asthma severity, luminal eosinophilia and age at onset. Thorax. 2012;67(12):1061. doi:10.1136/thoraxjnl-2012-201634

17. Ying S, Robinson DS, Meng Q, et al. Enhanced expression of eotaxin and CCR3 mRNA and protein in atopic asthma. Association with airway hyperresponsiveness and predominant co-localization of eotaxin mRNA to bronchial epithelial and endothelial cells. Eur $J$ Immunol. 2010;27 (12):3507-3516.

18. Chupp GL, Lee CG, Jarjour N, et al. A chitinase-like protein in the lung and circulation of patients with severe asthma. $N$ Engl $J$ Med. $2007 ; 357$ (20):2016-2027. doi:10.1056/NEJMoa073600

19. Gomez JL, Yan X, Holm CT, et al. Characterisation of asthma subgroups associated with circulating YKL-40 levels. Eur Respir J. 2017;50 (4):1700800. doi:10.1183/13993003.00800-2017

20. Roslind A, Johansen JS. YKL-40: a novel marker shared by chronic inflammation and oncogenic transformation. In: Kozlov SV, editor. Inflammation and Cancer: Methods and Protocols: Volume 1: Experimental Models and Practical Approaches. Totowa, NJ: Humana Press; 2009:159-184.

21. Ilmarinen P, Tuomisto LE, Niemelä O, et al. YKL-40 and adult-onset asthma: elevated levels in clusters with poorest outcome. $J$ Allergy Clin Immunol Pract. 2019;7(7):2466-2468.e3. doi:10.1016/j.jaip.2019.03.043

22. Wang J, Lv H, Luo Z, et al. Plasma YKL-40 and NGAL are useful in distinguishing ACO from asthma and COPD. Respir Res. 2018;19(1):1-10. doi:10.1186/s12931-018-0755-6

23. Nakajima H, Hirose K. Role of IL-23 and Th17 cells in airway inflammation in asthma. Immune Netw. 2010;10(1):1-4. doi:10.4110/in.2010.10.1.1

24. Wakashin H, Hirose K, Maezawa Y, et al. IL-23 and Th17 cells enhance Th2-cell-mediated eosinophilic airway inflammation in mice. Am J Respir Crit Care Med. 2008;178(10):1023-1032. doi:10.1164/rccm.200801-086OC

25. Moreira AP, Cavassani KA, Ismailoglu UB, et al. The protective role of TLR6 in a mouse model of asthma is mediated by IL-23 and IL-17A. J Clin Invest. 2011;121(11):4420-4432. doi:10.1172/JCI44999

26. Elaidy SM, Essawy SS, Hussain MA, El-Kherbetawy MK, Hamed ER. Modulation of the IL-23/IL-17 axis by fenofibrate ameliorates the ovalbumin/lipopolysaccharide-induced airway inflammation and bronchial asthma in rats. Naunyn Schmiedebergs Arch Pharmacol. 2017;391 (3):309-321.

27. Lai T, Wu D, Li W, et al. Interleukin-31 expression and relation to disease severity in human asthma. Sci Rep. 2016;6:22835. doi:10.1038/ srep22835

28. Neuper T, Neureiter D, Sarajlic M, et al. IL-31 transgenic mice show reduced allergen-induced lung inflammation. Eur J Immunol. 2020;51 (1):191-196. doi:10.1002/eji.202048547

29. Perrigoue JG, Li J, Zaph C, et al. IL-31-IL-31R interactions negatively regulate type 2 inflammation in the lung. J Exp Med. $2007 ; 204(3): 481-487$.

30. Huang J, Yue H, Jiang T, et al. IL-31 plays dual roles in lung inflammation in an OVA-induced murine asthma model. Biol Open. 2019;8(1). doi:10.1242/bio.036244

31. Corren J. Role of Interleukin-13 in Asthma. Curr Allergy Asthma Rep. 2013;13(5):415-420. doi:10.1007/s11882-013-0373-9

32. Collison A, Li J, Pereira de Siqueira A. Tumor necrosis factor-related apoptosis-inducing ligand regulates hallmark features of airways remodeling in allergic airways disease. Am J Respir Cell Mol Biol. 2014;51(1):86-93. doi:10.1165/rcmb.2013-0490OC

33. Weckmann M, Collison A, Simpson JL, et al. Critical link between TRAIL and CCL20 for the activation of TH2 cells and the expression of allergic airway disease. Nat Med. 2007;13(11):1308. doi:10.1038/nm1660

34. Robertson NM, Rosemiller M, Lindemeyer RG, Steplewski A, Zangrilli JG, Litwack G. TRAIL in the airways. Vitam Horm. $2004 ; 67(67): 149-167$.

35. Corrigan CJ, Wang W, Meng Q. Allergen-induced expression of IL-25 and IL-25 receptor in atopic asthmatic airways and late-phase cutaneous responses. J Allergy Clin Immunol. 2011;128(1):116-124. doi:10.1016/j.jaci.2011.03.043

36. Dolgachev V, Petersen BC, Budelsky AL, et al. Pulmonary IL-17E (IL-25) production and IL-17RB+ myeloid cell-derived Th2 cytokine production are dependent upon stem cell factor-induced responses during chronic allergic pulmonary disease. J Immunol. 2009;183(9):5705-5715. doi:10.4049/jimmunol.0901666 
37. Wang YH, Angkasekwinai P, Lu N, et al. IL-25 augments type 2 immune responses by enhancing the expansion and functions of TSLPDC-activated Th2 memory cells. J Exp Med. 2007;204(8):1837-1847. doi:10.1084/jem.20070406

38. Hong HY, Chen F-H, Sun Y-Q, et al. Local IL-25 contributes to Th2-biased inflammatory profiles in nasal polyps. Allergy. 2018;73(2):459-469. doi:10.1111/all.13267

\section{Publish your work in this journal}

The Journal of Asthma and Allergy is an international, peer-reviewed open-access journal publishing original research, reports, editorials and commentaries on the following topics: Asthma; Pulmonary physiology; Asthma related clinical health; Clinical immunology and the immunological basis of disease; Pharmacological interventions and new therapies. The manuscript management system is completely online and includes a very quick and fair peer-review system, which is all easy to use. Visit http://www.dovepress.com/testimonials.php to read real quotes from published authors.

Submit your manuscript here: https://www.dovepress.com/journal-of-asthma-and-allergy-journal 